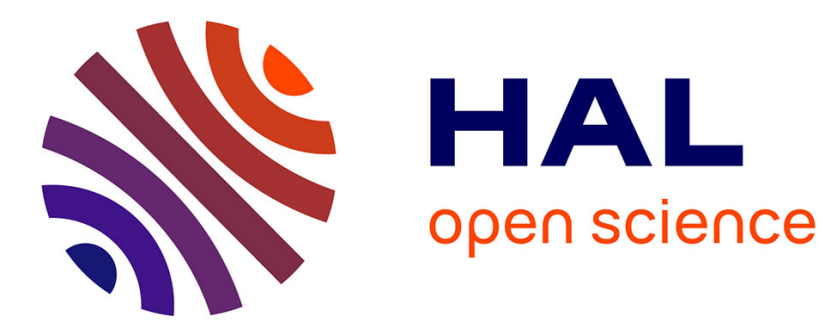

\title{
Channeled spectrum in the transmission of phononic crystal waveguides
}

Yan-Feng Wang, Tingting Wang, Jun-Wei Liang, Yue - Sheng Wang, Vincent

Laude

\section{- To cite this version:}

Yan-Feng Wang, Tingting Wang, Jun-Wei Liang, Yue - Sheng Wang, Vincent Laude. Channeled spectrum in the transmission of phononic crystal waveguides. Journal of Sound and Vibration, 2018, 437, pp.410-421. hal-02130172

\section{HAL Id: hal-02130172 \\ https://hal.science/hal-02130172}

Submitted on 15 May 2019

HAL is a multi-disciplinary open access archive for the deposit and dissemination of scientific research documents, whether they are published or not. The documents may come from teaching and research institutions in France or abroad, or from public or private research centers.
L'archive ouverte pluridisciplinaire HAL, est destinée au dépôt et à la diffusion de documents scientifiques de niveau recherche, publiés ou non, émanant des établissements d'enseignement et de recherche français ou étrangers, des laboratoires publics ou privés. 


\title{
Channeled spectrum in the transmission of phononic crystal waveguides
}

\author{
Yan-Feng Wang ${ }^{\mathrm{a}, \mathrm{b}}$, Ting-Ting Wang ${ }^{\mathrm{b}, \mathrm{c}}$, Jun-Wei Liang ${ }^{\mathrm{b}}$, Yue-Sheng Wang ${ }^{\mathrm{a}, \mathrm{b}}$, Vincent Laude ${ }^{\mathrm{c}}$ \\ ${ }^{a}$ School of Mechanical Engineering, Tianjin University, 300350 Tianjin, China \\ ${ }^{b}$ Institute of Engineering Mechanics, Beijing Jiaotong University, 100044 Beijing, China \\ ${ }^{c}$ Institut FEMTO-ST, Université de Franche-Comté, CNRS, F-25030 Besançon Cedex, France
}

\begin{abstract}
Waveguiding in a phononic crystal (PC) can be achieved along either linear line defects or a sequence of cavities, for frequencies belonging to a complete bandgap. When waves are coupled inside a PC waveguide, modulations in the frequency transmission are generally observed, leading to the formation of a channeled spectrum. We show that the channeled spectrum results from the interference of forward and backward guided Bloch waves. We first theoretically develop a Bloch wave interference model. Then, we consider the case of linear waveguides and of coupled-resonator waveguides formed in a 2D square PC composed of water cylinders in mercury. The transmission properties of waveguides with different length and defect distribution are examined. In all cases, the observed channeled spectra are well explained by the theoretical model. This work is relevant to the design of new acoustic and elastic wave devices.
\end{abstract}

Keywords: Phononic crystal, phononic crystal waveguide, channeled spectrum

\section{Introduction}

As a kind of artificially periodic functional composite, phononic crystals (PCs) have attracted a lot of attention since their proposal in 1993 [1]. One unique property of PCs is to exhibit bandgaps for certain frequency ranges, where wave propagation is fully prohibited. Thus, they have direct applications to sound insulation $[2,3,4]$ and vibration reduction [5, 6, 7], for instance. In addition, PCs can also present peculiar properties caused by strong dispersion at passing frequencies [8].

When periodicity is broken, defect states are generated, where waves are either localized in the cavity (point defects) or confined along the waveguide (line defects). Linear lines of defects, as the most commonly used guidance mechanism, have received a wide attention $[9,10]$. Many different waveguides have been formed in 2D [11, 12, 13] and 3D [14] PCs, PC slabs [15] with holes [14, 16] or pillars [17, 18], and for surface waves on semi-infinite PCs [19, 20], as well as in phoxonic crystals [21]. Both numerical simulations and experimental measurements were conducted

Email addresses: wangyanfeng@bjtu.edu.cn (Yan-Feng Wang), vincent.laude@femto-st.fr (Vincent Laude)
$[10,15,16,22,23]$ and were found to be in good agreement. One alternative guidance mechanism is using evanescent couplings along a linear chain of defect cavities or resonators inside the channel $[24,25,26]$, as inspired by coupled-resonator optical waveguides in photonic crystals [27]. In this case, the band structure can be changed by varying the coupling strength of the resonators through their separation. Single mode operation is also allowed.

Another guidance mechanism that has attracted much attention recently incorporates the additional property of topological protection and is based on a classical analog to the quantum Hall effect [28]. Topological phononic waveguides have for instance been demonstrated recently in elastic plates [29, 30,31]. Though we do not consider explicitly topological waveguides in the following, we suggest that the theory we develop applies as well to the description of their spectral transmission.

Whatever the guidance mechanism, it has been recognized from the beginning that the transmission of PC waveguides shows oscillations of the transmission as a function of frequency [9]. However, the nature of these oscillations has remained mostly unexplained. A similar phenomenon, the channeled spectrum, has long been known in guided-wave optics. For integrated optical 


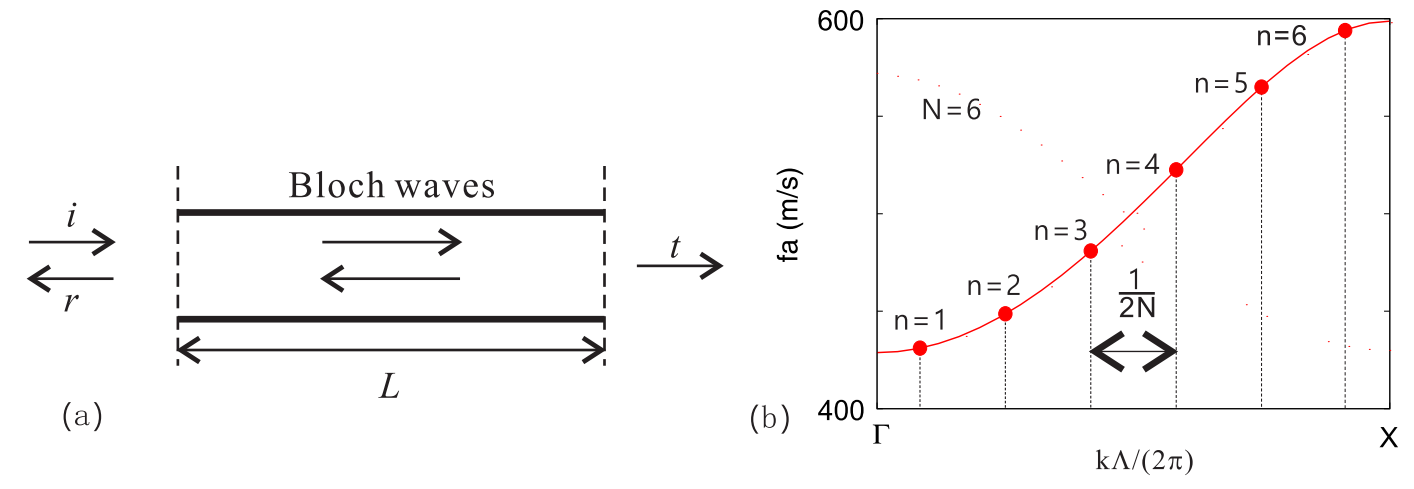

Figure 1: (a) Simplified model of transmission through a single-mode periodic waveguide at a single frequency. (b) Graphical construction of the channeled spectrum from the dispersion relation of the infinite waveguide for the particular cases $\theta=-\pi$ and $\theta=0$.

waveguides, for instance, the channeled spectrum is a classical technique for the characterization of dispersion properties and group delay dispersion $[32,33]$. A similar technique is also useful in the characterization of optical fibers [34].

The channeled spectrum in homogeneous waveguides results from the interference of forward propagating guided waves with the backward propagating waves generated by internal reflections, especially at the ends of the waveguide. In this paper, this description is extended to periodic waveguides, for which the channeled spectrum is considered as resulting from the interference of forward and backward guided Bloch waves. A theoretical model is developed that predicts accurately the distribution of frequencies at which maxima and minima of transmission occur, together with the pressure distribution at those frequencies. In order to illustrate the model, we consider the specific case of single Bloch wave propagation in either linear waveguides or coupled-resonator acoustic waveguides (CRAWs). The dispersive characteristics of the channeled spectrum are shown to depend mostly on the length of the PC waveguide and on the dispersion relation of the considered guided Bloch wave. The effect of viscous damping on the channeled transmission spectrum is finally investigated.

\section{Model}

In this section, a theoretical model is proposed to provide a physical explanation for the appearance of frequencies at which transmission maxima and minima occur, similar to the channeled spectrum in optics [35]. For a single-mode PC waveguide in the considered frequency range, the fundamental guided mode is a propagation channel for waves traveling to the right or to the left, as depicted in Fig. 1(a). The time-harmonic pressure field inside the waveguide can then be written as the superposition of a left-traveling Bloch wave, $p_{l}(x, y)$, with a right-traveling Bloch wave, $p_{r}(x, y)$,

$$
p(\omega ; x, y)=\alpha p_{r}(x, y) e^{-l k(\omega) x}+\beta p_{l}(x, y) e^{+l k(\omega) x} .
$$

In this equation, $x$ is a curvilinear coordinate along the waveguide axis and $y$ is a transverse coordinate. The functions $p_{l}(x, y)$ and $p_{r}(x, y)$ have periodicity $\Lambda$ along $x$ and satisfy $p_{l}(\Lambda-x, y)=p_{r}^{*}(x, y)$. They depend in principle on frequency, though we don't explicit this dependence here. The complex coefficients $\alpha$ and $\beta$ are to be determined. $k(\omega)$ is the Bloch wavenumber along the waveguide axis. Since we consider periodic waveguides with one direction of periodicity, the Bloch wavevector indeed reduces to a single wavenumber. Note that $k(\omega)$ can be obtained by inverting the dispersion relation $\omega(k)$ of the waveguide, with $k$ considered a continuous variable, as we perform in Section 3. It is also important to note that the two guided Bloch waves propagate unperturbed and that they only convert to one another at the ends of the waveguide. Hence, the model only considers a waveguide of finite length $L$ and periodicity is not directly apparent because it is already embedded in the guided Bloch waves.

By continuity of pressure, the transmission in amplitude at the exit of the waveguide can be determined as $t(\omega)=p(\omega ; L, 0)$, or

$$
t(\omega)=\alpha p_{r}(L, 0) e^{-l k(\omega) L}+\beta p_{l}(L, 0) e^{+l k(\omega) L},
$$

where $L=N \Lambda$ is the length of the waveguide and $N$ is the number of the periodic unit cells. We further assume that the $x$-periodic functions $p_{l}(x, y)$ and $p_{r}(x, y)$ are normalized such that $\left|p_{l}(0,0)\right|=\left|p_{l}(\Lambda, 0)\right|=1$ and $\left|p_{r}(0,0)\right|=\left|p_{r}(\Lambda, 0)\right|=1$. Any residual phase can then 
be absorbed in the coefficients $\alpha$ and $\beta$, and we finally have without loss of generality

$$
t(\omega)=\alpha e^{-\imath k(\omega) L}+\beta e^{+\imath k(\omega) L} .
$$

The transmission in intensity is then

$$
|t(\omega)|^{2}=|\alpha|^{2}+|\beta|^{2}+2|\alpha \beta| \cos (2 k(\omega) L-\theta),
$$

with $\theta=\operatorname{Arg}\left(\alpha \beta^{*}\right)$ a phase angle dependent on the particular superposition of guided Bloch waves.

Transmission maxima and minima are obtained when

$$
\frac{\mathrm{d}|t(\omega)|^{2}}{\mathrm{~d} \omega}=0
$$

Neglecting the possible dependence of $\alpha$ and $\beta$ on frequency, maxima are obtained when $2 k(\omega) L=\theta$ modulo $2 \pi$, or for a discrete sequence of frequencies and wavenumbers such that

$$
k\left(\omega_{n}\right) \Lambda=\frac{\theta}{2 N}+\frac{n}{N} \pi .
$$

As a result, frequencies at which transmission is maximum can be directly inferred from the dispersion relation of waveguide, as depicted in Fig. 1(b). It is noted that there are $N$ maxima given by Eq. (6) between points $\Gamma$ and $\mathrm{X}$ of the first Brillouin zone when $\theta \neq 0$. However, when $\theta=0$, there are $N-1$ maxima since the Bloch waves at high symmetry points $\Gamma$ and $X$ can hardly be excited, as we argue later. Similarly, frequencies at which transmission is minimum are obtained for

$$
k\left(\omega_{n+1 / 2}\right) \Lambda=\frac{\theta}{2 N}+\frac{n+1 / 2}{N} \pi .
$$

As follows from Eqs. (6-7), transmission maxima and minima are sampled uniformly along the $k$-axis with the step $\delta k=\frac{\pi}{N \Lambda}$ so that their density increases in proportion to the length of the waveguide. The frequencies of transmission maxima and minima have a variable distribution, or free spectral range. Indeed, their local separation is proportional to the group velocity, according to

$$
\delta \omega \approx v_{g}(k) \delta k=v_{g}(k) \frac{\pi}{N \Lambda}
$$

where the group velocity can be directly obtained from the dispersion relation as

$$
v_{g}(k)=\frac{\mathrm{d} \omega}{\mathrm{d} k} .
$$

When $k$ is close to the high symmetry points $\Gamma$ and $\mathrm{X}$, the group velocity tends to zero and the frequency spacing of transmission maxima and minima reduces. The present model is valid for any monomodal phononic crystal waveguide, as we illustrate next.

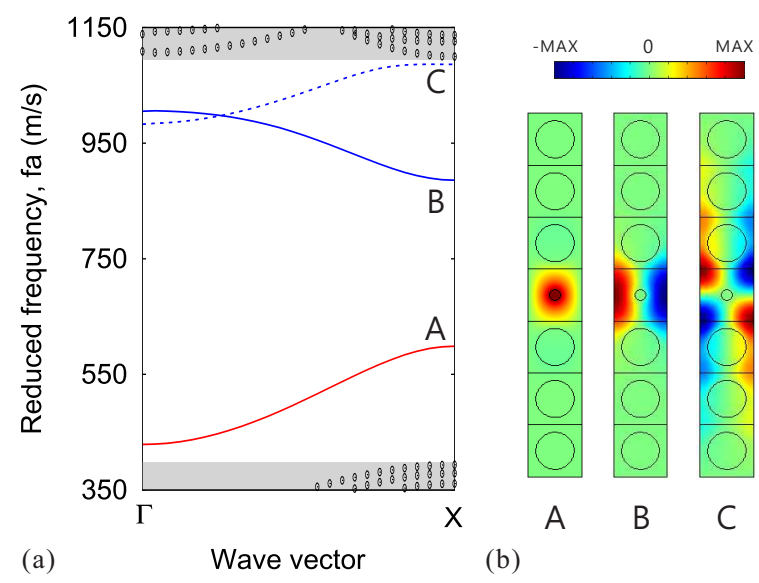

Figure 2: Dispersion relation of an infinitely linear waveguide with cavity radius $r / a=0.1$. (a) Band structure for a $7 \times 1$ super-cell of the square PC of water cylinders in mercury is presented in the $\Gamma \mathrm{X}$ direction of the first Brillouin zone. The gray areas indicate the passing frequency ranges. Three guided modes exist inside the complete bandgap and are labelled A, B and C. (b) Pressure distributions at the $\mathrm{X}$ point of the first Brillouin zone are presented for the three guided modes. The color scale represents the amplitude from blue for negative to red for positive values.

\section{Channeled transmission spectrum in linear waveguides}

As a direct implementation of the previous model, we select a 2D square PC composed of water cylinders embedded in mercury [36]. The dispersion relation of a linear waveguide with infinite length is first considered. Material parameters used here are $\rho=1025 \mathrm{~kg} / \mathrm{m}^{3}$, $c=1531 \mathrm{~m} / \mathrm{s}$ for water, and $\rho=13600 \mathrm{~kg} / \mathrm{m}^{3}, c=1450 \mathrm{~m} / \mathrm{s}$ for mercury. The filling fraction is 0.4 . In the absence of frequency-dependent loss, the PC can be scaled arbitrarily and its dispersion relation can be represented as reduced frequency $f a$ versus reduced wavenumber $k a$, with $f$ the frequency, $k$ the wavenumber, and $a$ the lattice constant. The perfectly periodic PC possesses a complete bandgap for $398 \mathrm{~m} / \mathrm{s}<f a<1095 \mathrm{~m} / \mathrm{s}$.

A linear waveguide is formed by introducing a line defect. Within the line defect, the radius of the water cylinders is changed to $r / a=0.1$. Fig. 2(a) illustrates the dispersion relation of the waveguide in the $\Gamma-X$ direction of the irreducible Brillouin zone. Finite element method [8] is used for all numerical simulations. Three guided modes are generated within the complete bandgap, in the frequency range of $429 \mathrm{~m} / \mathrm{s}<f a<599$ $\mathrm{m} / \mathrm{s}$ for Bloch wave A, $886 \mathrm{~m} / \mathrm{s}<f a<1005 \mathrm{~m} / \mathrm{s}$ for Bloch wave B and $983 \mathrm{~m} / \mathrm{s}<f a<1086 \mathrm{~m} / \mathrm{s}$ for Bloch wave $C$. Their pressure distributions at the $\mathrm{X}$ point are displayed in Fig. 2(b). Each guided mode has a special pattern of pressure distribution. The pressure amplitude 


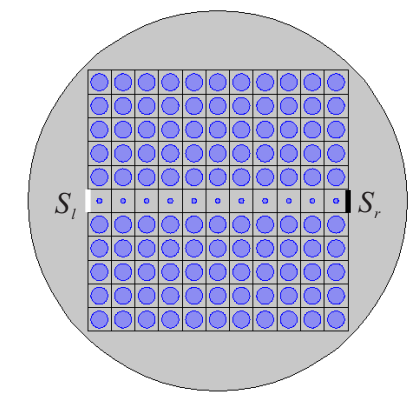

(a)

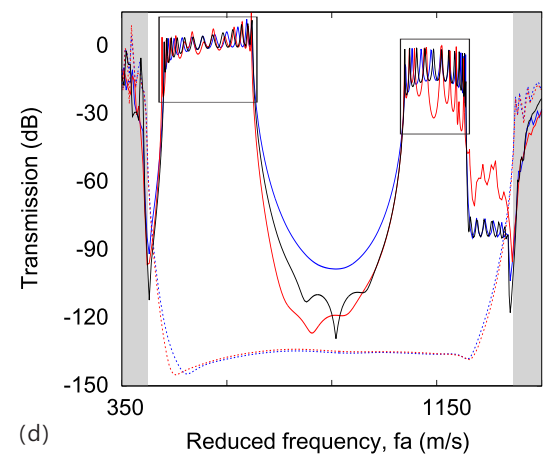

(b)
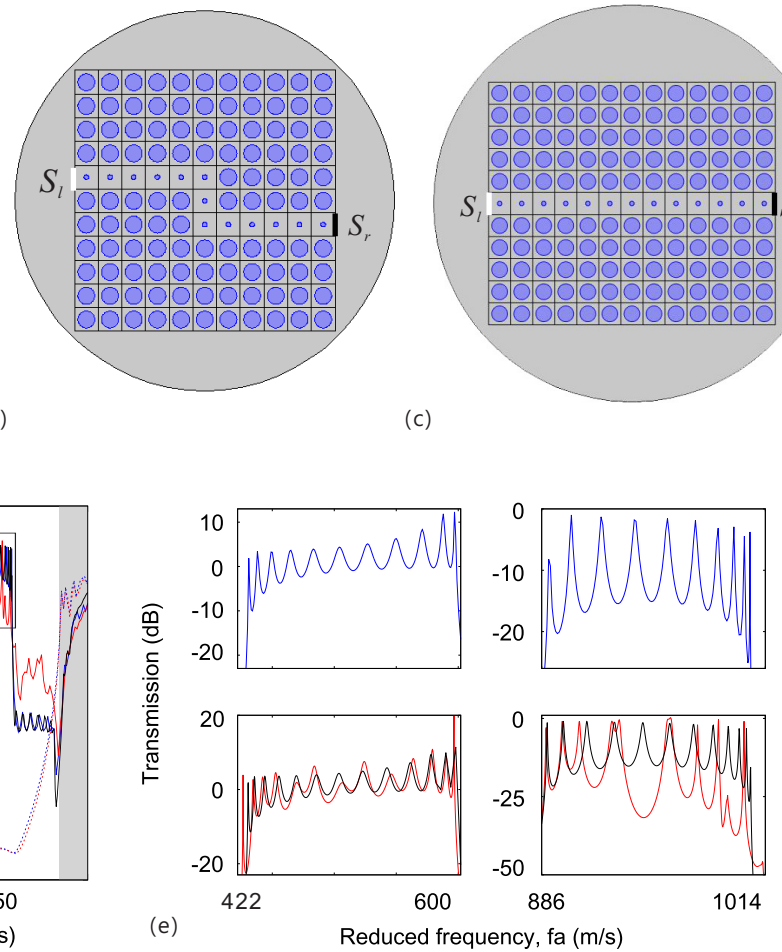

Figure 3: Cross-sections of linear waveguides arranged in three different circuits inside finite square PCs and the related transmission. The length $L$ of a waveguide is an integer multiple of the lattice constant $a$. We consider (a) a straight waveguide with $L=11 a$ (LW1), (b) a waveguide with 2 turns and $L=13 a(\mathrm{LW} 2)$, and (c) a straight waveguide with $L=13 a(\mathrm{LW} 3)$. The wave sources used for transmission computation are marked by white lines. The transmitted wave is detected along the black lines on the other side of the waveguide. (d) Transmission through waveguides LW1, LW2 and LW3 are shown with blue, red, and black solid lines, respectively. The dashed lines represent transmission through the perfect PC, with source and detector placed as in the case of waveguides LW1 and LW2. (e) For clarity, transmissions around $\mathrm{fa}=500 \mathrm{~m} / \mathrm{s}$ and $\mathrm{fa}=1000 \mathrm{~m} / \mathrm{s}$ are zoomed.

Table 1: Frequencies at which transmission is maximum for different waveguides in the lower or upper passing frequencies of Fig. 3(d). The units of reduced frequency, $f a$, are $\mathrm{m} / \mathrm{s}$.

\begin{tabular}{|c|c|c|c|c|c|c|c|c|c|c|c|c|c|}
\hline order & 1 & 2 & 3 & 4 & 5 & 6 & 7 & 8 & 9 & 10 & 11 & 12 & 13 \\
\hline \multirow[t]{2}{*}{ LW1 } & 431.2 & 438.2 & 449.4 & 464.6 & 483.2 & 504.2 & 526.8 & 549.8 & 571 & 587.8 & 597.4 & & \\
\hline & 1005.2 & 1002 & 996 & 987 & 974 & 958 & 939.4 & 920.2 & 902.8 & 890.4 & & & \\
\hline \multirow[t]{2}{*}{ LW2 } & 426.4 & 434.6 & 442.2 & 452.8 & 469.6 & 484 & 506.4 & 524.2 & 546 & 563.8 & 579.2 & 590.4 & 596.6 \\
\hline & 1012.4 & 1001.8 & 992.4 & 987.2 & 975.2 & 959.2 & 958.4 & 930.4 & 926.2 & 907.6 & 897.8 & 888.6 & \\
\hline \multirow[t]{2}{*}{ LW3 } & 430.6 & 435.6 & 444 & 455.4 & 469.4 & 485.6 & 503.8 & 523 & 542.6 & 561.4 & 578 & 590.8 & 597.8 \\
\hline & 1005.6 & 1003.2 & 999 & 982.8 & 984.2 & 973 & 959.4 & 943.8 & 927.6 & 911.8 & 898.4 & 889.2 & \\
\hline
\end{tabular}

at points A and B decays rapidly away from the linear defect in the transverse direction, owing to the existence of the complete bandgap, so guided modes are well confined to the linear defect. Bloch wave $\mathrm{C}$ is strongly influenced by the passing bands right above the bandgap and its confinement is weak.

The appearance of channeled transmission spectra is intimately linked to the finite length of a waveguide and to the termination conditions. Here, we consider linear waveguides with different length and number of turns, as depicted in Fig. 3. The finite structures have $11 \times 11$ unit cells in Figs. 3(a) and (b), and $13 \times 11$ unit cells in Fig. 3(c). Three different linear waveguides are considered: (a) a straight waveguide with length $L=11 a$, (b) a waveguide with length $L=13 a$ and 2 turns, and (c) a straight waveguide with length $L=13 a$. In the following, they are referred to as the LW1, the LW2 and the LW3 waveguides, respectively. 


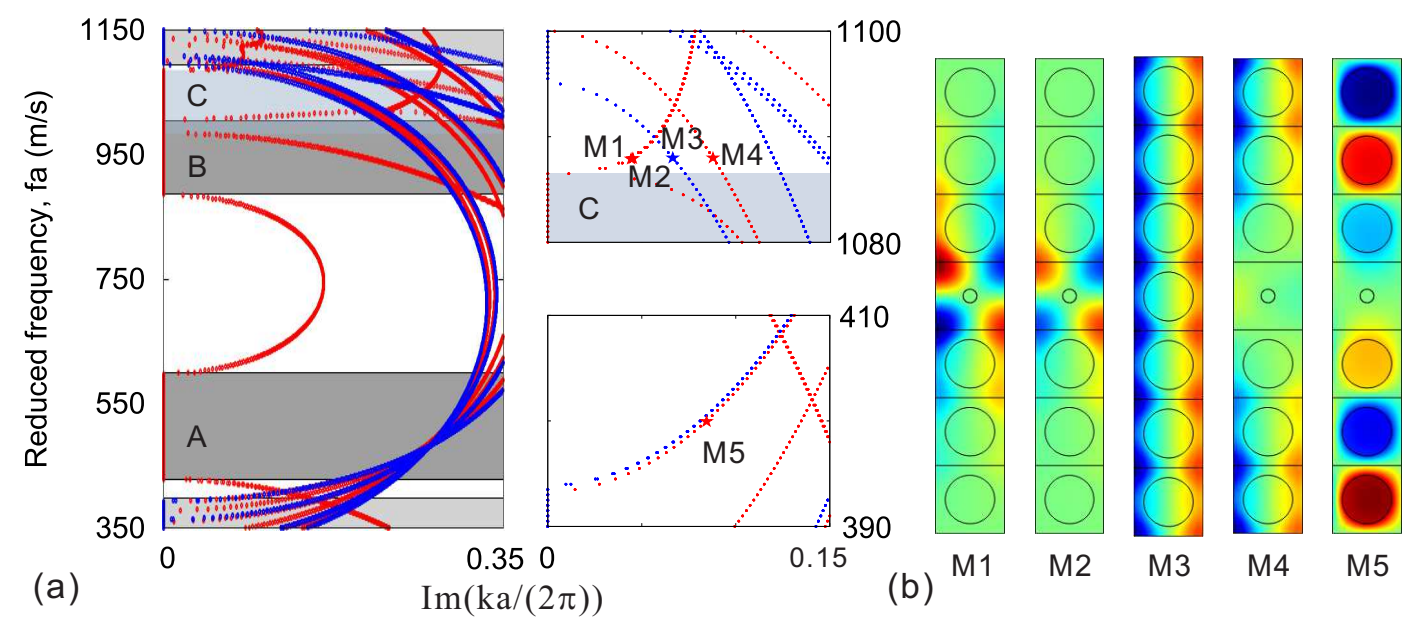

Figure 4: Imaginary part of the complex band structure of the linear waveguide (red dots). The dark-gray and slate gray regions in panel (a) correspond to the frequency ranges of the three guided waves in Fig. 2(a). The blue dots represent the results for the bare PC computed by a $7 \times 1$ super-cell. For clarity, band structures around $f a=400 \mathrm{~m} / \mathrm{s}$ and $f a=1090 \mathrm{~m} / \mathrm{s}$ are zoomed. The pressure distributions in the linear waveguide and in the bare PC at the marked points in panel (a) are illustrated in panel (b).

One characteristic of waveguides is their transmission for acoustic waves, which can be evaluated as follows. The finite PC is placed in a homogeneous mercury region. A radiation boundary condition is set on the outer boundary, closing the computation domain and minimizing reflections. A line source for acoustic waves with unit amplitude $p_{0}=1$ is positioned at the left end $S_{l}$ of the waveguide. The width of the source segment is exactly one lattice constant $a$. By sweeping the reduced frequency in the frequency range of interest, the transmission $T(f a)$ in decibels units can be estimated as

$$
T(f a)=20 \log _{10}\left(\frac{\int_{S_{r}}|p| \mathrm{d} s}{\int_{S_{l}}\left|p_{0}\right| \mathrm{d} s}\right)
$$

where $p$ is the pressure along the right end $S_{r}$ of the waveguide. It is worth noting that the transmission as defined above can be larger than unity without violating energy conservation [37].

Fig. 3(c) shows transmissions for the different waveguides as a function of reduced frequency. For comparison, transmissions for the perfect PC, i.e. with no defect, are also plotted. Two passing frequency ranges come up clearly inside the complete bandgap. They coincide with the dispersion relations of the waveguides in Fig. 2(a), except for Bloch wave $\mathrm{C}$ in the frequency range of $1005 \mathrm{~m} / \mathrm{s}<f a<1086 \mathrm{~m} / \mathrm{s}$. Bloch wave $\mathrm{C}$ is actually a deaf mode [38], since the pressure distribution at point $\mathrm{C}$ shown in Fig. 2(b) is anti-symmetric with respect to the direction of the wave vector (the hor- izontal axis). Such a mode can thus not be excited by an incident plane wave. However, evanescent Bloch waves with the right symmetry can still be excited, as we argue later, and result in a small transmission. The upper passing range is thus identified with Bloch wave $\mathrm{B}$. The lower one corresponds to Bloch wave A. In the extent of passing frequency ranges, only small differences in transmission exist for the different circuits. This indicates that waveguides with an arbitrary number of turns can be designed. Transmission is generally very low for frequencies in-between passing bands and is then dominated by the total length of the waveguide. The smaller transmission is thus found for the longer waveguide.

For comparison, transmissions for bare PCs are also calculated and plotted in Fig. 3. It is observed that the transmission through waveguides can be smaller than through the perfect PC. This is owing to the reconstruction of the complex band structure, especially the imaginary part of the Bloch wavenumber shown in Fig. 4. The detailed calculation process can be found in Refs [8, 39]. It is known that the minimum imaginary part of the Bloch wavenumber dominates the transmission [40]. For the bare PC, the minimum imaginary part is always non-zero in the bandgap. When linear defects are introduced, its value changes a lot. There are even some frequency ranges where it is larger than for the bare PC (see the zoomed panels). The pressure distributions of evanescent Bloch waves at the marked points in Fig. 4(a) are shown in Fig. 4(b). For the linear waveguide, the modes at points M1 and M2 of the imaginary bands are asymmetric with respect to the $x$-axis. So they 

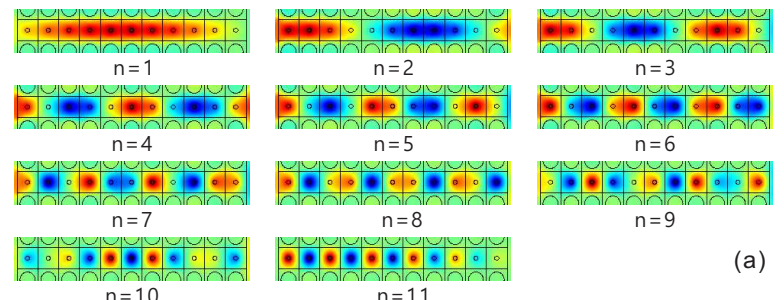

$n=10$

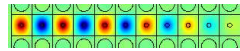
$n=11$

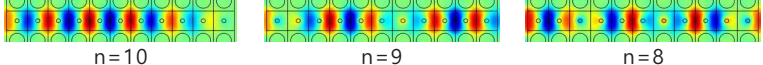

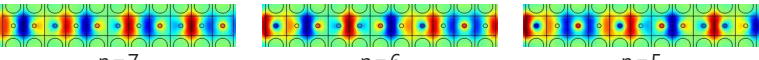

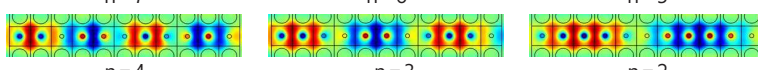 मै० $n=3 \quad n=2$ $n=1$

Figure 5: Pressure distribution of the straight waveguide (LW1, $L=$ $10 a$ ) at the resonant peaks of the (a) lower or (b) upper passing frequencies listed in Table 1. The number of pressure oscillations are shown below the field maps.
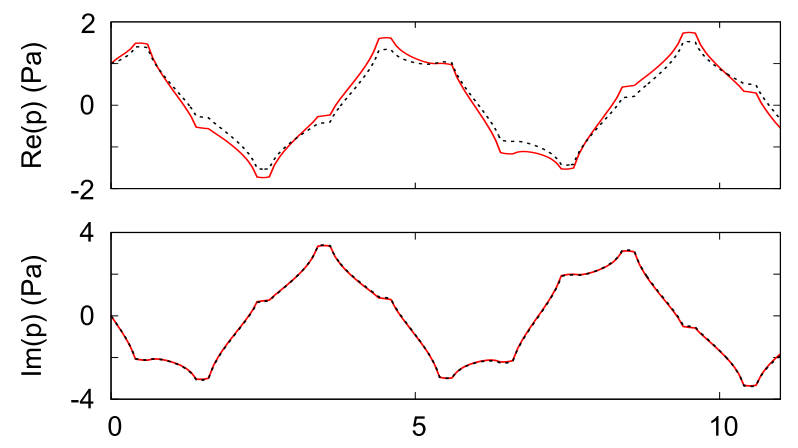

(a)

$\mathrm{x} / \mathrm{a}$
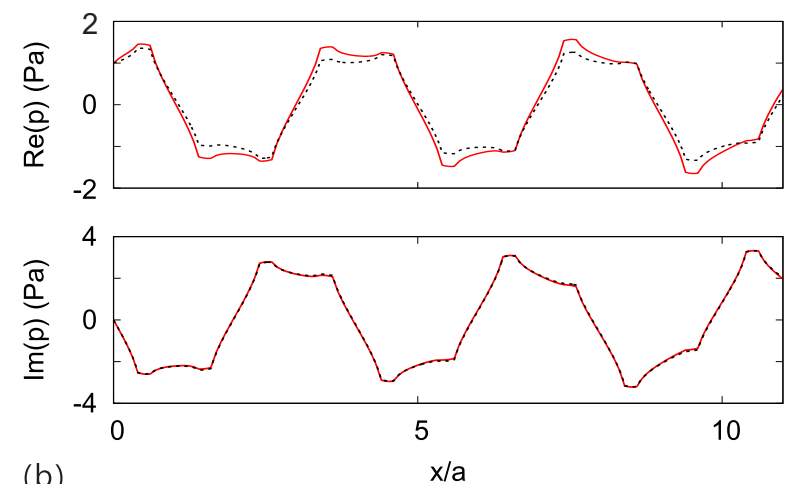

(b)

Figure 6: Numerical (solid line) and predicted (dashed line) pressure distribution along the central line of the straight linear waveguide LW1 in Fig. 5(a) for (a) $\mathrm{n}=5(\alpha=-1.40+1.37 l, \beta=2.38+1.76 l)$ and (b) $\mathrm{n}=6(\alpha=-1.08+1.60 l, \beta=2.18+1.94 l)$.

can not be excited in the simulation, similarly to Bloch
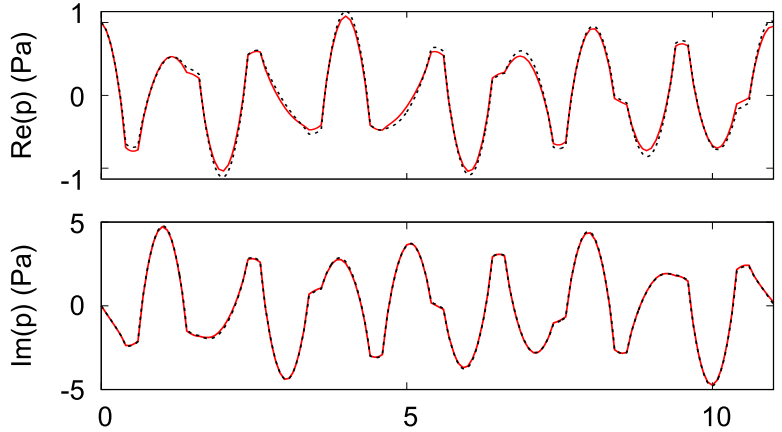

(a)

$\mathrm{x} / \mathrm{a}$
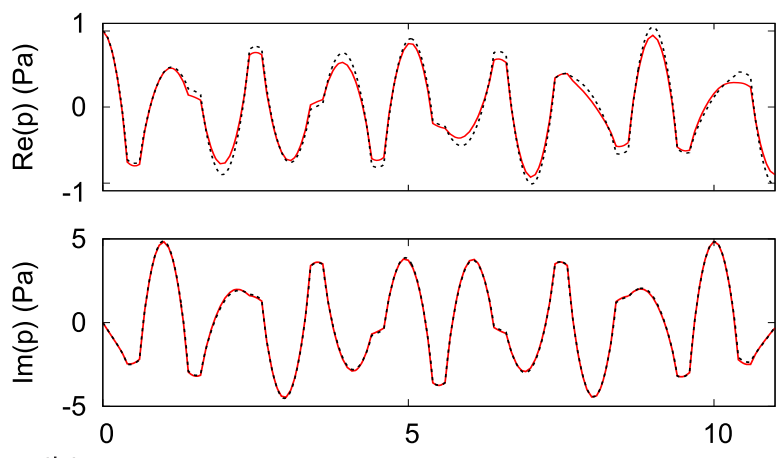

(b)

$\mathrm{x} / \mathrm{a}$

Figure 7: Numerical (solid line) and predicted (dashed line) pressure distribution along the central line of the straight linear waveguide LW1 in Fig. 5(b) for (a) $\mathrm{n}=5(\alpha=1.37-0.91 \imath, \beta=-0.43-0.50 \imath)$ and (b) $\mathrm{n}=6(\alpha=1.20-1.08 \imath, \beta=-0.35-0.51 \imath)$.

wave C. Mode M3 for the bare PC, and modes M4 and M5 for the linear waveguide can be excited. Clearly, the latter two modes have larger imaginary parts and so they decay faster than mode M3. A smaller transmission for the waveguides is then expected, in coincidence with the results in Fig. 3(c).

The lower and the upper passing frequency ranges support single mode propagation and large maximum transmission. Fig. 3(d) shows a close-up view at transmission within the lower and upper passing frequency ranges. A pattern of successive maxima and minima appears in the transmission spectra. Table 1 reports the frequencies with maximum transmissions for the three considered waveguides. Waveguides with the same length but different number of turns, such as LW2 and LW3, have the same number and positions of extremal frequencies, though slight differences in amplitude. Bloch wave A has a doubly symmetric shape but is slightly 'compressed' by periodicity towards the left and the right. Then the coupling between adjacent defect is slightly asymmetric along the $x$-axis (left/right) compared to the $y$-axis (up/down). The number of max- 
ima is inferred to be $N$ for the lower passing frequency and $N-1$ for the upper frequency range, though in both case the length of the waveguide is $L=N a$. We have checked that this apparent discrepancy is not owing to the frequency resolution in the computation, but can rather be attributed to the value of the phase angle $\theta$ in Eq. (6). There are indeed generally $N$ positive values of $k\left(\omega_{n}\right) \Lambda$ for $\theta \neq 0$ and $N+1$ possible values if $\theta=0$. However, when a particular value falls close to 0 or $\pi$, the group velocity tends to zero: the Bloch wave will not be efficiently excited and hence will not appear in the transmission. As a result, there can be either $N$ or $N-1$ transmission peaks.

Pressure fields at the observed maxima of the straight waveguide LW1 with $L=11 a$ are shown in Fig. 5(a) for the lower passing frequency and Fig. 5(b) for the upper passing frequency. As $n$ increases, the number of spatial oscillations along the waveguide is seen to increase, in accordance with Eq. (6). In general, the envelopes of the guided wave fields indeed show sinusoidal variations, as described by Eq. (1).

The model of Eq. (1) can only be approximate since evanescent Bloch waves are not taken into account [41]. Evanescent Bloch waves are expected to be excited at the two ends of the waveguide and to decay rapidly with distance. Figs. 6 and 7 show the real and imaginary parts of the pressure distribution for the lower or upper frequency range, along the central line of the straight waveguide for maxima $n=5$ and $n=6$. In the lower passing frequency, 5 or 6 oscillations are clearly observed in the envelopes of the pressure distribution. While for the upper frequency range, the oscillations are too faint to be distinguished. The pressure distributions obtained by using Eq. (1) after fitting $\alpha$ and $\beta$ are also given in Figs. 6 and 7. There is a good overall agreement between computed and fitted pressure distributions.

\section{Channeled transmission spectrum in coupled- resonator acoustic waveguides}

In this section, we consider coupled-resonator acoustic waveguides (CRAWs) formed by sequences of defect cavities. The separation between adjacent cavities is $\Lambda$, which is an integral number of lattice constants. We select $\Lambda=2 a$ in this section, and the supercell is shown in Fig. 8(a). A cavity is introduced by replacing one cylinder of water with mercury. By sweeping the wavevector in the $\Gamma-X$ direction of the first Brillouin zone, the dispersion relation of the CRAW is obtained and illustrated in Fig. 8(b). The reduced wavenumber, $k \Lambda /(2 \pi)$, is used in the band structure. Three guided modes are formed within the complete bandgap. Their pressure distributions at the $\Gamma$ point are displayed in Fig. 8(c). The pressure distributions of the guided Bloch waves have the same symmetries as those in Fig. 2(b). Bloch wave F is a deaf mode, although it has a better confinement than Bloch wave C.

Next, we consider CRAWs with different lengths and number of turns defined in finite PCs, as shown in Figs. 9(a)-(d). Four different variations on the theme of CRAWs are considered: (a) a straight chain of 10 cavities, (b) a chain of 13 cavities and 2 turns, (c) a chain of 17 cavities and 8 turns, and (d) the same as (b) but for a straight chain. For simplicity, they are named CW1, CW2, CW3, and CW4, respectively.

Fig. 9(e) shows transmissions for the different circuits. For comparison, transmissions for the perfect PC are also presented. Two passing frequency ranges appear clearly inside the complete bandgap, coinciding with the CRAW dispersion bands in Fig. 8(b). Transmissions for different circuits have slight differences in the extent of passing frequency ranges. This indicates that the CRAW principle can be applied to design rather arbitrary waveguides, i.e. waveguides with arbitrary number of turns. In the upper passing frequency range, the transmissions for different circuits have large variations that strongly depend on the considered waveguide. Indeed, the more turns along the waveguide, the larger the variations. We do not attribute these complications to the presence of two guided modes in this frequency range, since Bloch wave $\mathrm{F}$ is deaf, but rather to the modal shape of Bloch wave E (shown in Fig. 8(c)) that is not as compatible with turns as the modal shape of Bloch wave D. Bloch waves D and A have a similar doubly symmetric shape, so they can almost couple equally to 4 different directions. The only difference is that Bloch wave A is slightly more 'compressed' by periodicity, as we noted before, so its coupling is slightly asymmetric along the horizontal and vertical directions. This is further proved by the comparison of the transmission for waveguides with same length but different turns in Figs. 3(e) and 9(f). In the following, we will correspondingly focus our attention on the lower passing frequency range that supports single mode propagation and large maximum transmission. Low transmissions are observed for frequencies in-between passing bands, similar to those in Fig. 3. They are generally determined by the total length of the waveguide. Longer CRAWs generally have smaller transmission. It is also noted that there is a spurious resonance appearing above the lower passing frequency range. Its generation is directly related to the excitation of a localized resonance close to the line source, as the pressure distri- 


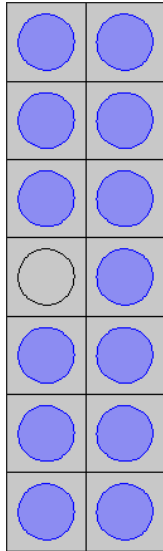

(a)

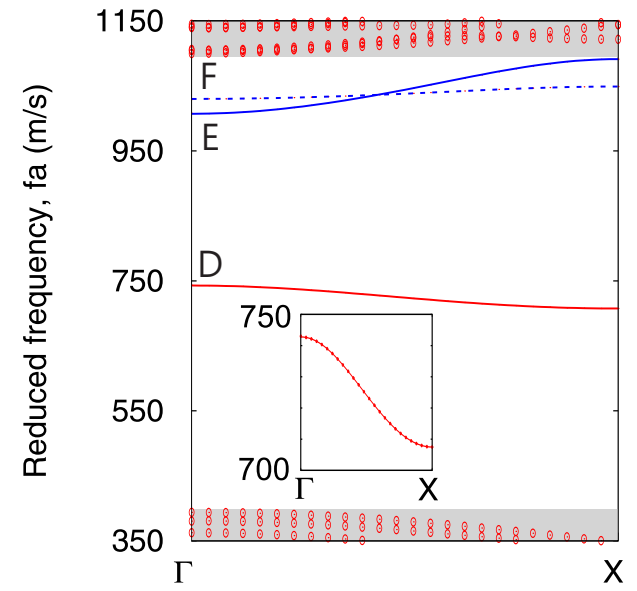

(b)

$\mathrm{k} \Lambda /(2 \pi)$

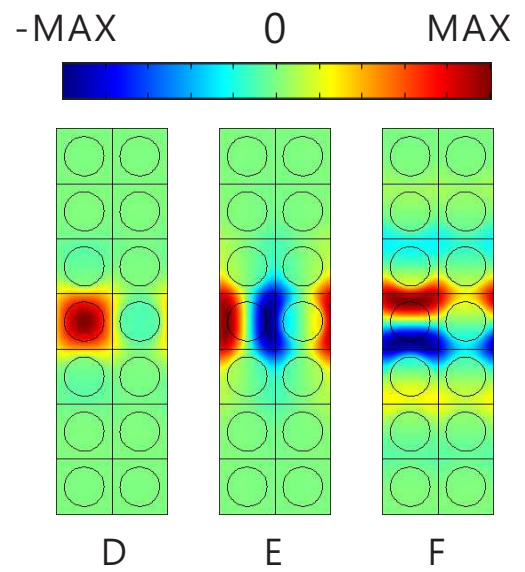

(c)

Figure 8: Band structure of a coupled-resonator acoustic waveguide with cavity separation $\Lambda=2 \mathrm{a}$. (a) A $7 \times 2$ super-cell of the square PC of water cylinders in mercury (b) The dispersion relation is presented in the $\Gamma X$ direction of the first Brillouin zone. The gray areas indicate the passing frequencies. Scatterers and lines represent the numerical and fitted results, respectively. (c) Pressure distributions for the three guided modes are presented at dispersion points D, E and F. The color scale extends symmetrically from blue for negative to red for positive values.

bution shown in the inset of Fig. 9(e) shows. Given the very low transmission at this local resonance, it can be safely ignored.

Fig. 9(f) shows a close-up view at transmission within the lower passing frequency range. A pattern of successive maxima and minima appears in the transmission spectra. Table 2 reports the frequencies with maximum transmissions for the four considered waveguides, presented in decreasing order in accordance with the dispersion relation of Bloch wave D in Fig. 8(b). It is observed that waveguides with the same length (CW2 and CW4) have the same number and positions of maxima of transmission, similar to the results for linear waveguides. Moreover, the effect of the turns is lower compared to that for the linear waveguides, owing to the higher confinement of the CRAWs. The number of maxima is inferred to be $N-1$ (as shown in Fig. 1(b)) if the length of the waveguide is $L=N \Lambda$, in accordance with Eq. (6) when $\theta=0$. Indeed, for $n=0$ ( $\Gamma$ point $)$ and $n=N$ (X point), the guided Bloch waves have zero group velocity and are not excited by a source placed outside the waveguide.

Pressure fields in the straight CRAW CW1 with $L=$ $10 \Lambda$ are shown in Fig. 10 for the 9 observed maxima. Similar to the linear waveguides, the number of spatial oscillations along the waveguide is seen to increase with an increase in $n$. Fig. 11 shows the real and imaginary parts of the pressure distribution along the central line of the straight waveguide for maxima $n=5$ and $n=6$, as well as the results predicted by using Eq. (1) after fitting $\alpha$ and $\beta$. A good overall agreement between computed and fitted pressure distributions is again observed. In general, it can be concluded that the channeled transmission spectrum is well explained by the simple model presented in Section 2.

In practice, acoustic wave damping has distinct effects on the transmission through a waveguide. The frequency-dependent viscosity of water and mercury [42] can be taken into account by using a viscous fluid model [43]. Viscous losses effectively increase with the square of frequency with this model. The effects of damping on the transmission spectrum for different lattice constants $a$ is shown in Fig. 12, in the case of the straight waveguide. Changing the lattice constant amounts to tuning the operating frequency range. It is observed that the influence of viscosity remains limited if the lattice constant is larger than $2 \mu \mathrm{m}$, i.e. for operating frequencies lower than about $300 \mathrm{MHz}$. The effect of viscosity however increases rapidly for smaller lattice constants. Viscous effect in passing frequency ranges is more apparent as compared to their outside, because guided waves have small group velocities, and the spatial decay on propagation is known to vary inversely with the group velocity [44]. In the limit of large viscous damping, the spectral oscillations in the channeled spectrum tend to be washed out, indicating a quenching of the interference of forward and backward guided Bloch waves. 


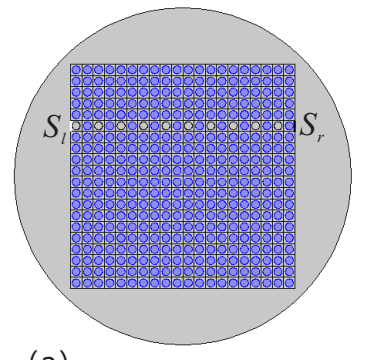

(a)

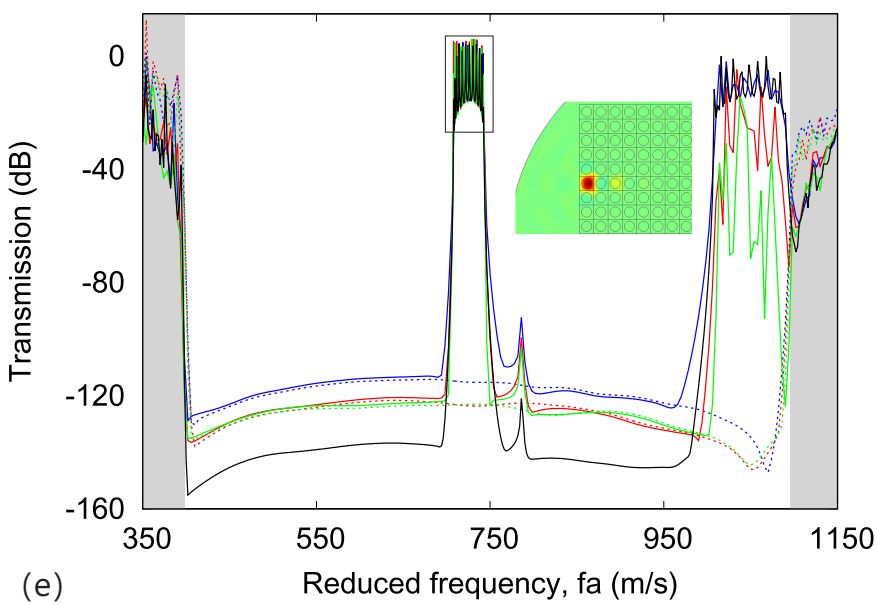

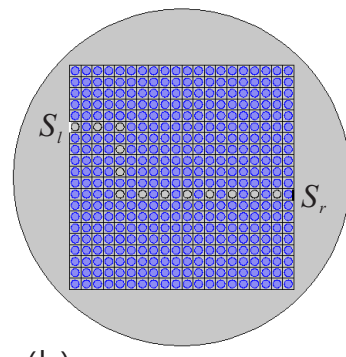

(b)

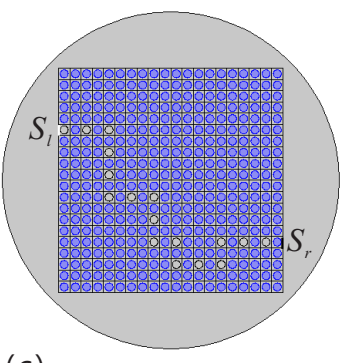

(c)

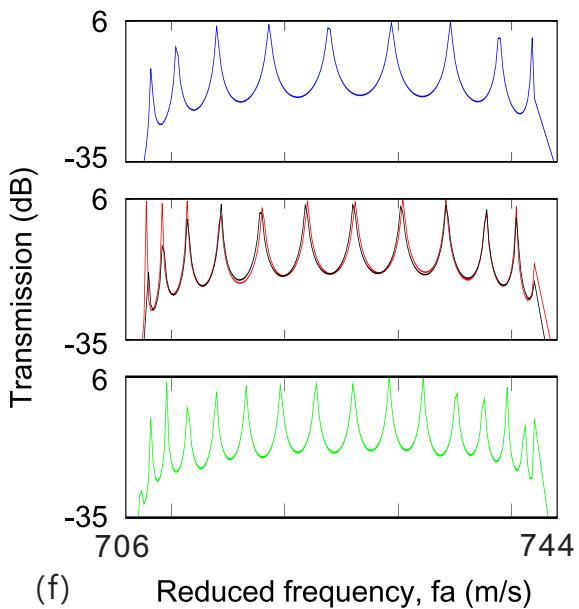

Figure 9: Cross-sections of coupled-resonator acoustic waveguides arranged in different circuits inside finite PCs and the related transmission. The length $L$ of a waveguide is an integer multiple of the cavity separation $\Lambda=2 a$. We consider (a) a straight waveguide with $L=10 \Lambda$ (CW1), (b) a waveguide with 2 turns and $L=13 \Lambda(\mathrm{CW} 2)$, (c) a waveguide with 8 turns and $L=17 \Lambda(\mathrm{CW} 3)$, and (d) a straight waveguide with $L=13 \Lambda$ (CW4). The wave sources used for transmission computation are marked by white lines. Transmitted waves are detected at the black lines. (e) Transmissions for CW1, CW2, CW3 and CW4 are shown by the blue, red, green and black lines, respectively. The dashed lines represent transmission through the perfect PC, with source and detector placed as in the case of waveguides. The inset shows the pressure distribution at $f a=786 \mathrm{~m} / \mathrm{s}$ for the straight waveguide. (f) For clarity, transmission around $f a=725 \mathrm{~m} / \mathrm{s}$ is zoomed.

Table 2: Frequencies at which transmission is maximum for different waveguides in the lower passing frequency of Fig. 9. The units of reduced frequency, $f a$, are $\mathrm{m} / \mathrm{s}$

\begin{tabular}{lllllllllllllllll}
\hline order & 1 & 2 & 3 & 4 & 5 & 6 & 7 & 8 & 9 & 10 & 11 & 12 & 13 & 14 & 15 & 16 \\
\hline CW1 & 741.8 & 738.8 & 734.6 & 729.4 & 723.8 & 718.6 & 714 & 710.4 & 708.2 & & & & & \\
CW2 & 742 & 740.4 & 737.8 & 734.2 & 730.4 & 726.2 & 722 & 718 & 714.4 & 711.4 & 709.2 & 707.8 & & \\
CW3 & 742 & 741.2 & 739.6 & 737.4 & 735.2 & 732.2 & 729.2 & 726 & 722.8 & 719.6 & 716.6 & 714 & 711.4 & 709.6 & 708.2 & 707.4 \\
CW4 & 742 & 740.4 & 737.8 & 734.2 & 730.2 & 726 & 721.8 & 717.8 & 714.4 & 711.4 & 709.2 & 707.8 & & \\
\hline
\end{tabular}

\section{Conclusions}

In this paper, we have discussed the appearance of channeled transmission spectra in phononic crystal waveguides. We have proposed a model that predicts the pressure distribution in the waveguide and the shape of the channeled spectrum. The model is based on the interference of forward and backward guided Bloch waves. For illustration, we have considered acoustic wave propagation in single-mode acoustic waveguides. The waveguides were formed by introducing either line defects or linear chains of cavities in a sonic crystal of water rods in mercury. It was observed that channeled spectra are dependent on the length of the waveguide but are almost independent of the circuit details, including the number of turns. Theoretical results agree well with numerical transmissions and pressure distributions. Finally, the effect of fluid viscosity on the transmission were evaluated as a function of the lattice constant. Quenching of the channeled spectrum with increasing damping is predicted. 


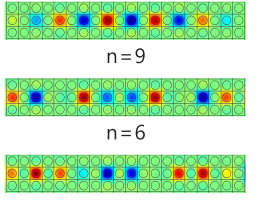

$n=3$
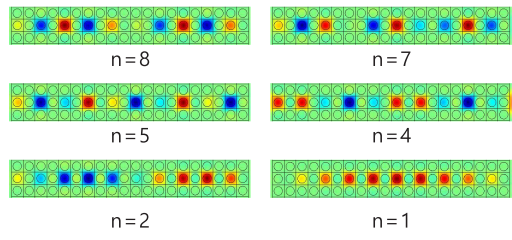

Figure 10: Pressure distribution of the straight CRAW $(\mathrm{CW} 1, L=$ $10 \Lambda)$ at the resonant peaks listed in Table 2. The number of pressure oscillations are shown below the field maps.
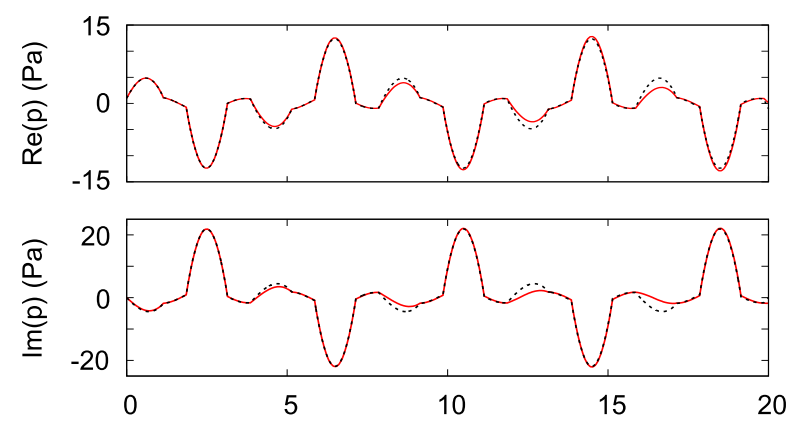

(a)

$\mathrm{x} / \mathrm{a}$
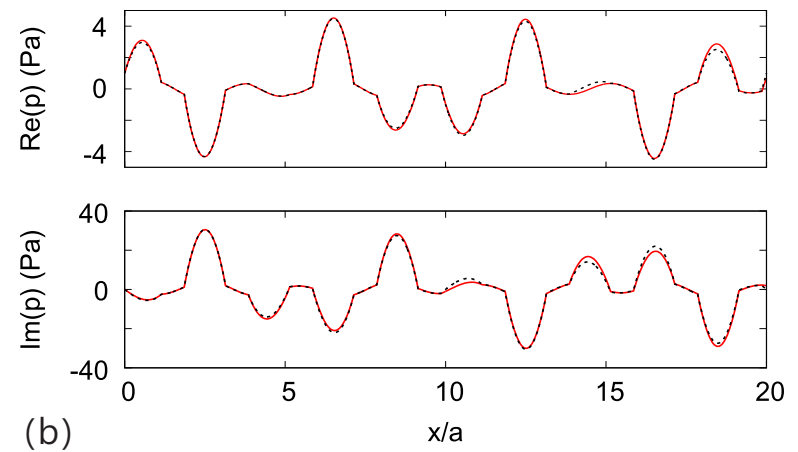

Figure 11: Numerical (solid line) and predicted (dashed line) pressure distribution along the central line of the straight CRAW CW1 in Fig. 10 for (a) $\mathrm{n}=5(\alpha=-0.81-0.72 \imath, \beta=1.20+0.42 \imath)$ and (b) $\mathrm{n}=6$ $(\alpha=-1.24-0.35 \imath, \beta=1.50-0.02 \imath)$. Real and imaginary parts of the pressure are both illustrated in the upper or lower part, respectively.

It could be extended to multimode PC waveguides, though the shape of the channeled spectrum would obviously be less simple. If one wants to remove the occurrence of maxima and minima in the transmission spectrum, care should be taken to engineer the terminations of the waveguide to minimize reflections, for instance by using tapering techniques similar to electromagnetic and optical waveguides [45].

\section{Acknowledgments}

The authors would like to acknowledge financial support from the National Natural Science Foundation of

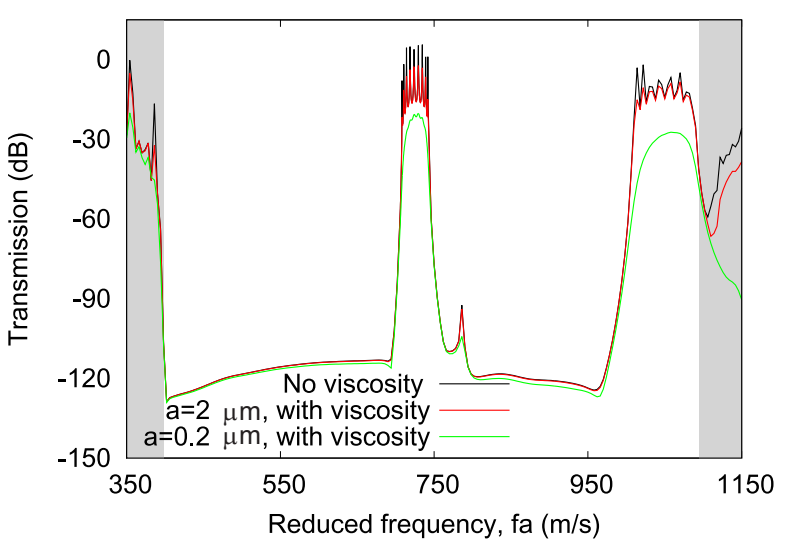

Figure 12: Influence of viscous damping in liquids on the transmissions of the straight CRAW CW1 in Fig. 9(a) for different lattice constants.

China (11702017,11532001 and 11642023), the Fundamental Research Funds for the Central Universities (2018JBM033), and the Labex ACTION program (Contract No. ANR-11-LABX-0001-01).

\section{References}

[1] M. S. Kushwaha, P. Halevi, L. Dobrzyński, B. Djafari-Rouhani, Acoustic band structure of periodic elastic composites, Physical Review Letters 71 (13) (1993) 2022.

[2] C.-Y. Huang, J.-H. Sun, T.-T. Wu, A two-port ZnO/silicon Lamb wave resonator using phononic crystals, Applied Physics Letters 97 (3) (2010) 031913.

[3] S. Castiñeira Ibáñez, C. Rubio, V. Romero-García, J. V. Sánchez-Pérez, L. M. García-Raffi, Design, manufacture and charaterization of an acoustic barrier made of multi-phenomena cylinders scatterers arranged in a fractal-based geometry, Archives of Acoustics 37 (4) (2012) 455-462.

[4] F. Morandi, M. Miniaci, A. Marzani, L. Barbaresi, M. Garai, Standardised acoustic characterization of sonic crystals noise barriers: Sound insulation and reflection properties, Applied Acoustics 114 (2016) 294-306.

[5] D. Yu, J. Wen, H. Zhao, Y. Liu, X. Wen, Vibration reduction by using the idea of phononic crystals in a pipe-conveying fluid, Journal of Sound and Vibration 318 (2008) 193-205.

[6] Y. Pennec, J. O. Vasseur, B. Djafari-Rouhani, L. Dobrzyński, P. A. Deymier, Two dimensional phononic crystals: examples and applications, Surface Science Reports 65 (8) (2010) 229291.

[7] P. A. Deymier, Acoustic Metamaterials and Phononic Crystals, Springer, Berlin, 2013.

[8] V. Laude, Phononic Crystals: Artificial Crystals for Sonic, Acoustic, and Elastic Waves, Walter de Gruyter GmbH, Berlin, 2015.

[9] M. Kafesaki, M. M. Sigalas, N. García, Frequency modulation in the transmittivity of wave guides in elastic-wave band-gap materials, Physical Review Letters 85 (19) (2000) 4044.

[10] A. Khelif, A. Choujaa, S. Benchabane, B. Djafari-Rouhani, V. Laude, Guiding and bending of acoustic waves in highly confined phononic crystal waveguides, Applied Physics Letters 84 (22) (2004) 4400-4402. 
[11] M. Torres, F. M. de Espinosa, Ultrasonic band gaps and negative refraction, Ultrasonics 42 (1) (2004) 787-790.

[12] Y. Pennec, B. Djafari-Rouhani, J. O. Vasseur, H. Larabi, A. Khelif, A. Choujaa, S. Benchabane, V. Laude, Acoustic channel drop tunneling in a phononic crystal, Applied Physics Letters 87 (26) (2005) 261912.

[13] T. Miyashita, Acoustic defect-mode waveguides fabricated in sonic crystal: Numerical analyses by elastic finite-difference time-domain method, Japanese Journal of Applied Physics 45 (5S) (2006) 4440.

[14] H. Chandra, P. A. Deymier, J. O. Vasseur, Elastic wave propagation along waveguides in three-dimensional phononic crystals, Physical Review B 70 (5) (2004) 054302.

[15] F.-L. Hsiao, A. Khelif, H. Moubchir, A. Choujaa, C.-C. Chen, V. Laude, Waveguiding inside the complete band gap of a phononic crystal slab, Physical Review E 76 (5) (2007) 056601.

[16] M. Ghasemi Baboly, A. Raza, J. Brady, C. M. Reinke, Z. C. Leseman, I. El-Kady, Demonstration of acoustic waveguiding and tight bending in phononic crystals, Applied Physics Letters 109 (18) (2016) 183504.

[17] M. Oudich, M. B. Assouar, Z. Hou, Propagation of acoustic waves and waveguiding in a two-dimensional locally resonant phononic crystal plate, Applied Physics Letters 97 (19) (2010) 193503.

[18] Y. Jin, N. Fernez, Y. Pennec, B. Bonello, R. P. Moiseyenko, S. Hémon, Y. Pan, B. Djafari-Rouhani, Tunable waveguide and cavity in a phononic crystal plate by controlling whisperinggallery modes in hollow pillars, Physical Review B 93 (5) (2016) 054109.

[19] J.-H. Sun, T.-T. Wu, Propagation of surface acoustic waves through sharply bent two-dimensional phononic crystal waveguides using a finite-difference time-domain method, Physical Review B 74 (17) (2006) 174305.

[20] M. Addouche, M. A. Al-Lethawe, A. Elayouch, A. Khelif, Subwavelength waveguiding of surface phonons in pillars-based phononic crystal, AIP Advances 4 (12) (2014) 124303.

[21] T.-R. Lin, C.-H. Lin, J.-C. Hsu, Enhanced acousto-optic interaction in two-dimensional phoxonic crystals with a line defect, Journal of Applied Physics 113 (5) (2013) 053508.

[22] P. H. Otsuka, K. Nanri, O. Matsuda, M. Tomoda, D. M. Profunser, I. A. Veres, S. Danworaphong, A. Khelif, S. Benchabane, V. Laude, O. B. Wright, Broadband evolution of phononiccrystal-waveguide eigenstates in real- and k-spaces, Scientific Reports 3 (2013) 3351.

[23] M. Ghasemi Baboly, C. M. Reink, B. A. Griffin, I. EI-Kady, Z. C. Leseman, Acoustic waveguiding in a silicon carbide phononic crystals at microwave frequencies, Applied Physics Letters 112 (10) (2018) 103504

[24] R. Sainidou, N. Stefanou, A. Modinos, Linear chain of weakly coupled defects in a three-dimensional phononic crystal: A model acoustic waveguide, Physical Review B 74 (17) (2006) 172302.

[25] J. M. Escalante, A. Martínez, V. Laude, Dispersion relation of coupled-resonator acoustic waveguides formed by defect cavities in a phononic crystal, Journal of Physics D: Applied Physics 46 (47) (2013) 475301.

[26] Y.-F. Wang, T.-T. Wang, Y.-S. Wang, V. Laude, Reconfigurable phononic crystal circuits formed by coupled acoustoelastic resonators, Physical Review Applied 8 (2017) 014006.

[27] A. Yariv, Y. Xu, R. K. Lee, A. Scherer, Coupled-resonator optical waveguide: a proposal and analysis, Optics Letters 24 (11) (1999) 711-713.

[28] R. Süsstrunk, S. D. Huber, Observation of phononic helical edge states in a mechanical topological insulator, Science 349 (6243) (2015) 47-50.
[29] S. H. Mousavi, A. B. Khanikaev, Z. Wang, Topologically protected elastic waves in phononic metamaterials, Nature Communications 6 (2015) 8682.

[30] J. Vila, R. K. Pal, M. Ruzzene, Observation of topological valley modes in an elastic hexagonal lattice, Physical Review B 96 (13) (2017) 134307.

[31] M. Miniaci, R. Pal, B. Morvan, M. Ruzzene, Observation of topologically protected helical edge modes in Kagome elastic plates, arXiv preprint arXiv:1710.11556.

[32] M. N. Armenise, C. Canali, M. De Sario, A. Carnera, P. Mazzoldi, G. Celotti, Characterization of $\mathrm{TiO}_{2}, \mathrm{LiNb}_{3} \mathrm{O}_{8}$, and $\left(\mathrm{Ti}_{0.65} \mathrm{Nb}_{0.35}\right) \mathrm{O}_{2}$ compound growth observed during Ti:LiNbO 3 optical waveguide fabrication, Journal of Applied Physics 54 (11) (1983) 6223-6231.

[33] G. G. Bentini, M. Bianconi, M. Chiarini, L. Correra, C. Sada, P. Mazzoldi, N. Argiolas, M. Bazzan, R. Guzzi, Effect of low dose high energy $\mathrm{O}_{3+}$ implantation on refractive index and linear electro-optic properties in $\mathrm{X}$-cut $\mathrm{LiNbO}_{3}$ : Planar optical waveguide formation and characterization, Journal of Applied Physics 92 (11) (2002) 6477-6483.

[34] P. Le Boudec, T. Chartier, F. Sanchez, E. Delevaque, R. Leners, G. Stéphan, P.-L. François, Channeled spectrum of a fiber laser, Optics Letters 18 (5) (1993) 355-357.

[35] K. Oka, T. Kato, Spectroscopic polarimetry with a channeled spectrum, Optics Letters 24 (21) (1999) 1475-1477.

[36] M. S. Kushwaha, P. Halevi, Giant acoustic stop bands in twodimensional periodic arrays of liquid cylinders, Applied Physics Letters 69 (1) (1996) 31-33.

[37] Y.-F. Wang, Y.-S. Wang, V. Laude, Wave propagation in twodimensional viscoelastic metamaterials, Physical Review B 92 (10) (2015) 104110.

[38] V. Laude, S. Moiseyenko, R. P.and Benchabane, N. F. Declercq, Bloch wave deafness and modal conversion at a phononic crystal boundary, AIP Advances 1 (04) (2011) 041402.

[39] Y.-F. Wang, V. Laude, Y.-S. Wang, Coupling of evanescent and propagating guided modes in locally resonant phononic crystals, Journal of Physics D: Applied Physics 47 (47) (2014) 475502.

[40] V. Romero-García, J. V. Sánchez-Pérez, S. Castiñeira Ibáñez, L. M. Garcia-Raffi, Evidences of evanescent Bloch waves in phononic crystals, Applied Physics Letters 96 (12) (2010) 124102.

[41] V. Laude, Y. Achaoui, S. Benchabane, A. Khelif, Evanescent Bloch waves and the complex band structure of phononic crystals, Physical Review B 80 (9) (2009) 092301.

[42] R. E. Graves, B. M. Argrow, Bulk viscosity: past to present, Journal of Thermophysics and Heat Transfer 13 (3) (1999) 337342 .

[43] S. Temkin, Elements of Acoustics, Acoustical Society of America, West Barnstable, MA, 2001.

[44] V. Laude, J. M. Escalante, A. Martínez, Effect of loss on the dispersion relation of photonic and phononic crystals, Physical Review B 88 (22) (2013) 224302.

[45] D. Deslandes, K. Wu, Integrated microstrip and rectangular waveguide in planar form, IEEE Microwave and Wireless Components Letters 11 (2) (2001) 68-70. 